\title{
Probing the radar scattering cross-section for high-energy particle cascades in ice
}

\author{
Rasha Abbasi $^{a}$, John Belz ${ }^{a}$, Dave Besson ${ }^{b}$, Krijn D. de Vries $^{* c}$, Michael DuVernois ${ }^{d}$, \\ Kael Hanson ${ }^{d}$, Daisuke Ikeda ${ }^{e}$, Uzair Latif ${ }^{b}$, Joshua Macy ${ }^{b}$, John Matthews ${ }^{a}$, \\ Thomas Meures $^{d}$, Aongus O'Murchadha ${ }^{d}$, Steven Prohira ${ }^{b}$, Bokkyun Shin ${ }^{f}$, Gordon \\ Thomson $^{a}$, Simona Toscano ${ }^{c}$ \\ ${ }^{a}$ Physics And Astronomy, University of Utah, 201 South, Salt Lake City, UT 84112, U.S. \\ ${ }^{b}$ Kansas University Physics Department, 1082 Malott Hall, Lawrence KS, 66045, USA \\ ${ }^{c}$ Vrije Universiteit Brussel, Dienst ELEM, IIHE, Pleinlaan 2, 1050, Brussel, Belgium \\ ${ }^{d}$ Dept. of Physics and Wisconsin IceCube Particle Astrophysics Center, University of Wisconsin, \\ Madison, WI 53706, USA \\ ${ }^{e}$ ICRR, University of Tokyo, 5-1-5 Kashiwanoha, Kashiwa, Chiba 277-8522, Japan \\ ${ }^{f}$ Osaka City University, Osaka 558-8585, Japan \\ E-mail: krijndevries@gmail.com
}

\begin{abstract}
Recently the radar scattering technique to probe neutrino induced particle cascades above $\mathrm{PeV}$ energies in ice was investigated. The feasibility of the radar detection method was shown to crucially depend on several up to now unknown plasma properties, such as the plasma lifetime and the free charge collision rate. To determine these parameters, a radar scattering experiment was performed at the Telescope Array Electron Light Source facility, where a beam of highenergy electrons was directed in a block of ice. The induced ionization plasma was consequently probed using a radar detection set-up detecting over a wide frequency range from $200 \mathrm{MHz}$ up to $2 \mathrm{GHz}$. First qualitative results of this experiment will be presented.
\end{abstract}

35th International Cosmic Ray Conference -ICRC2017-

10-20 July, 2017

Bexco, Busan, Korea

${ }^{*}$ Speaker. 


\section{Introduction}

With the detection of the high-energy cosmic neutrino flux at energies above $100 \mathrm{TeV}$ [1], the IceCube neutrino observatory opened a new window to the universe. Since neutrinos are chargeless, and weakly interacting, they are able to travel cosmological distances without deflection or absorption. This makes the neutrino the only particle to probe the high-energy universe above $\mathrm{PeV}$ energies at large redshifts.

Nevertheless, at higher energies, the cosmic neutrino flux drops rapidly. Consequently, above several PeV, the IceCube neutrino detector runs out of statistics. To detect the cosmic neutrino flux at even higher energies, one has to cover an even larger volume than the cubic kilometer probed by IceCube. Due to its long attenuation length, in combination with the cost efficient detectors, the radio signal is ideal to probe such volumes. Even-though a neutrino induced particle cascade in ice emits a transient radio pulse, so-called, Askaryan emission, the detection threshold for this emission typically lies above $10^{17} \mathrm{eV}$ [2]. To probe this signal, several Askaryan radio detectors are currently under construction, where their current configurations already should be close to the single event detection threshold $[3,4,5]$.

It follows there is currently an energy gap between several $\mathrm{PeV}$ where IceCube runs low in statistics, and a few EeV where the Askaryan radio detectors become sensitive. To cover this gap, in $[6,7]$, the radar detection technique was proposed. By actively probing the plasma induced by the high-energy neutrino induced particle cascade in ice, it was shown that the energy threshold could be lowered to PeV energies [6]. Nevertheless, the feasibility of the method crucially depends on several so-far unknown plasma properties, such as its lifetime and free charge collision rate.

Therefore, an overall efficiency factor was introduced, leading to an effective radar scattering cross-section. To determine this cross-section, in January 2015, a beam test experiment was performed at the Telescope Array Electron Light Source (TA-ELS) facility [8]. During this experiment a first hint for a radar scatter was observed [9]. Nevertheless, the obtained data did not allow us to claim a radar scatter. To confirm and investigate this possible scatter, in December 2016, a second experiment was performed. In these proceedings, we describe the first qualitative results from this second experiment.

\section{The Telescope Array Electron Light Source facility}

The Telescope Array is the largest cosmic-ray air shower detector in the Northern hemisphere [10]. When an ultra-high-energy cosmic-ray interacts with Earth's atmosphere a secondary particle cascade is induced. The Telescope Array observatory detects both the particle footprint at Earth's surface, as well as the fluorescence light emitted by air molecules that were excited by the particle cascade. For the absolute calibration of the fluorescence telescopes, the TA-ELS facility was constructed $100 \mathrm{~m}$ away from one of the fluorescence telescopes. The TA-ELS directs a high-energy electron beam into the air, with a $0.5 \mathrm{~Hz}$ repetition rate. In its standard configuration, the beam length is roughly $1 \mu \mathrm{s}$, and consists of $10^{8}-10^{9}$ electrons with an average energy of $40 \mathrm{MeV}$ per electron, giving a total energy of $\sim 40 \mathrm{PeV}$. To have a higher peak intensity, this beam length can be adjusted down to roughly $10 \mathrm{~ns}$, which was the configuration used for the experiment described in this work. The electron beam itself consists out of multiple sub-bunches which are 
emitted at $350 \mathrm{ps}$ intervals, corresponding to $2.856 \mathrm{GHz}$. To monitor the beam, either a Faraday Cup or pick-up coils are used. The total accuracy on the energy calibration is estimated to be roughly $3 \%$.

\section{Experimental set-up}

For this experiment, two different radio antenna set-ups were used. The first set-up consisted out of broad-band Vivaldi Horn antennas, which are sensitive between $600 \mathrm{MHz}$ and $6 \mathrm{GHz}$. Nevertheless, as will be shown in this work, the sensitivity loss when shifting to lower frequencies is relatively weak, which allows us to detect down to $50 \mathrm{MHz}$. Besides the Vivaldi antennas, a second antenna set-up was used consisting of Log-Periodic Dipole Antennas (LPDAs), sensitive in the 10-300 MHz range.

Since we are interested in the radar detection technique to probe high-energy particle cascades in ice, a block of ice was placed at the beam exit (see Fig. 1). Furthermore, from measurements described in [11], and [12], we expect a temperature dependence on the free charge lifetime, which is expected to increase toward lower temperatures. Therefore, the ice was actively cooled, and its temperature was monitored. During the experiment the ice temperature was typically between $-20^{\circ} \mathrm{C}$ and $-40^{\circ} \mathrm{C}$ to mimic the ice conditions at the South Pole, which would provide the ideal location for a possible large scale in-nature experiment. Besides the data obtained with the ice target, also several measurements were performed while directing the beam into the air.

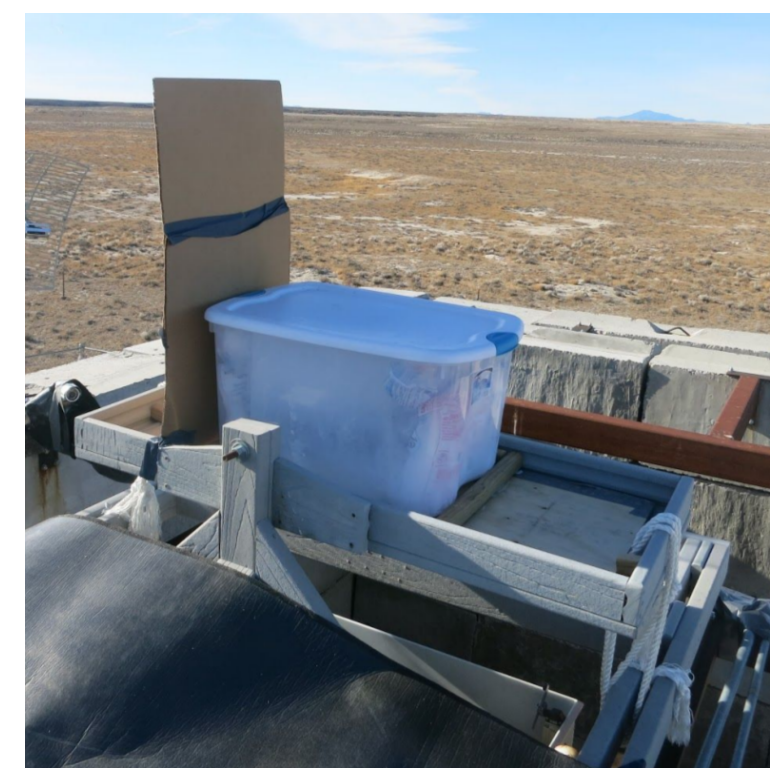

Figure 1: The block of ice located on top of the accelerator exit. The ice was actively cooled during the experiment.

\subsection{LPDA set-up}

The LPDA set-up used during this experiment consisted out of two EMCO LPDAs having roles of a transmitter and a receiver respectively (see Fig. 2). The distances of the transmitter 
and the receiver to the block of ice were $6.5 \mathrm{~m}$ and $6.3 \mathrm{~m}$ respectively, with the distance between the two antennas being $2.8 \mathrm{~m}$. Both antennas were operating at the same polarization, which could be altered during the experiment. Two probing schemes were applied for the radar scattering measurements. The first scheme consisted out of a pulsed emission, having $25 \mathrm{~ns}$ pulses with a dead time of $175 \mathrm{~ns}$ in between. The second scheme consisted out of a pure CW emission. The plasma was probed with transmission frequencies of $200 \mathrm{MHz}, 300 \mathrm{MHz}, 400 \mathrm{MHz}$ and $500 \mathrm{MHz}$. For each frequency the transmission power was varied to $-5 \mathrm{dBm},-10 \mathrm{dBm}$, and $-15 \mathrm{dBm}$.

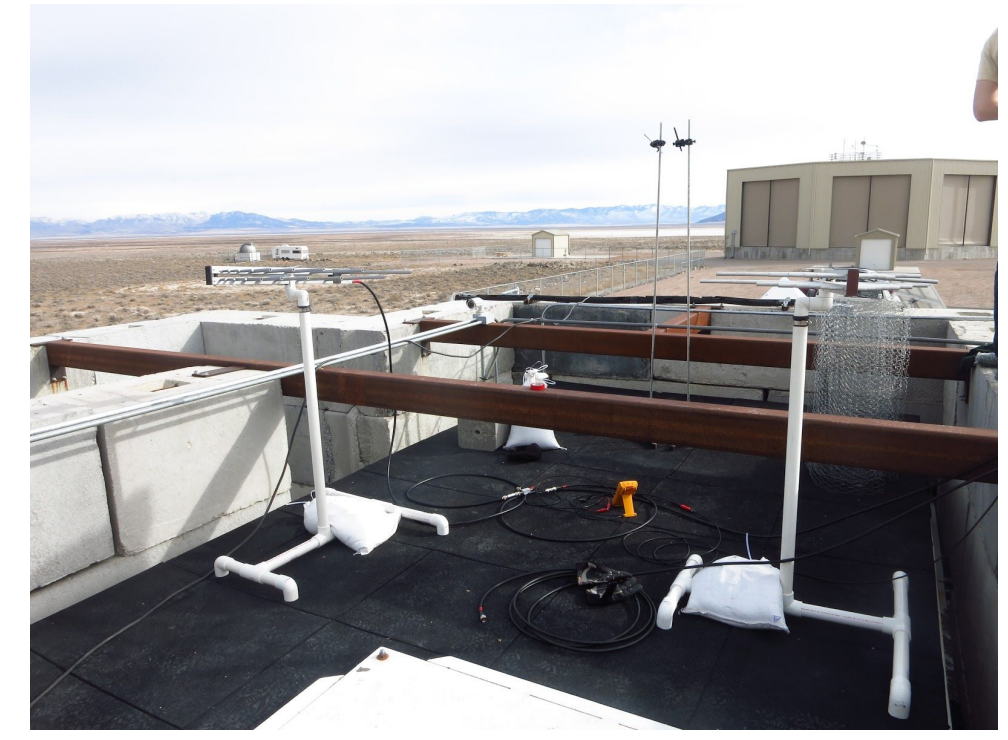

Figure 2: A front view of the LPDA set-up, consisting out of two EMCO LPDAs. During the experiment, the antennas were shielded by an RF absorbing screen.

\subsection{Vivaldi set-up}

The Vivaldi set-up consisted out of four $(600 \mathrm{MHz}-6 \mathrm{GHz})$ Vivaldi antennas located on the rooftop of the ELS (see Fig. 3). The relatively low signal loss below $600 \mathrm{MHz}$, allowed us to obtain data at frequencies down to $50 \mathrm{MHz}$. Besides the Vivaldi antennas, we also mounted four 1.4-3 GHz WiFibow antennas on a cross, which could be phased towards the block of ice. During the data-taking the metal parts visible in Fig. 3 were covered with RF absorbers. The signal chain for this set-up is shown in Fig. 4. The transmit signal is produced by the RF synthesizer, which generates a Continuous Wave $(\mathrm{CW})$ signal at a user specified frequency and signal strength. The generated signal was split in three channels. After splitting, one signal was led to the Vivaldi transmit antenna through a 150' LMR-400 co-axial cable, where it was amplified at the antenna $(+30$ $\mathrm{dB})$. Since we are searching for a scattered signal at the transmit frequency, the direct transmit signal observed by the receivers poses a large background. Therefore, the other two signal channels were directed through an attenuator and a phase shifter. The latter was done to mix these signals with the direct transmit signals observed by two of the Vivaldi receiver antennas (Rx), which allowed us to phase out this direct signal. The signals from the receiver antennas pass through a 75' LMR-400 co-axial cable, after which a low-pass filter was applied at $2.4 \mathrm{GHz}$ before they were mixed with the (out of phase) generated signal. After mixing, the signals were read out by a 10 

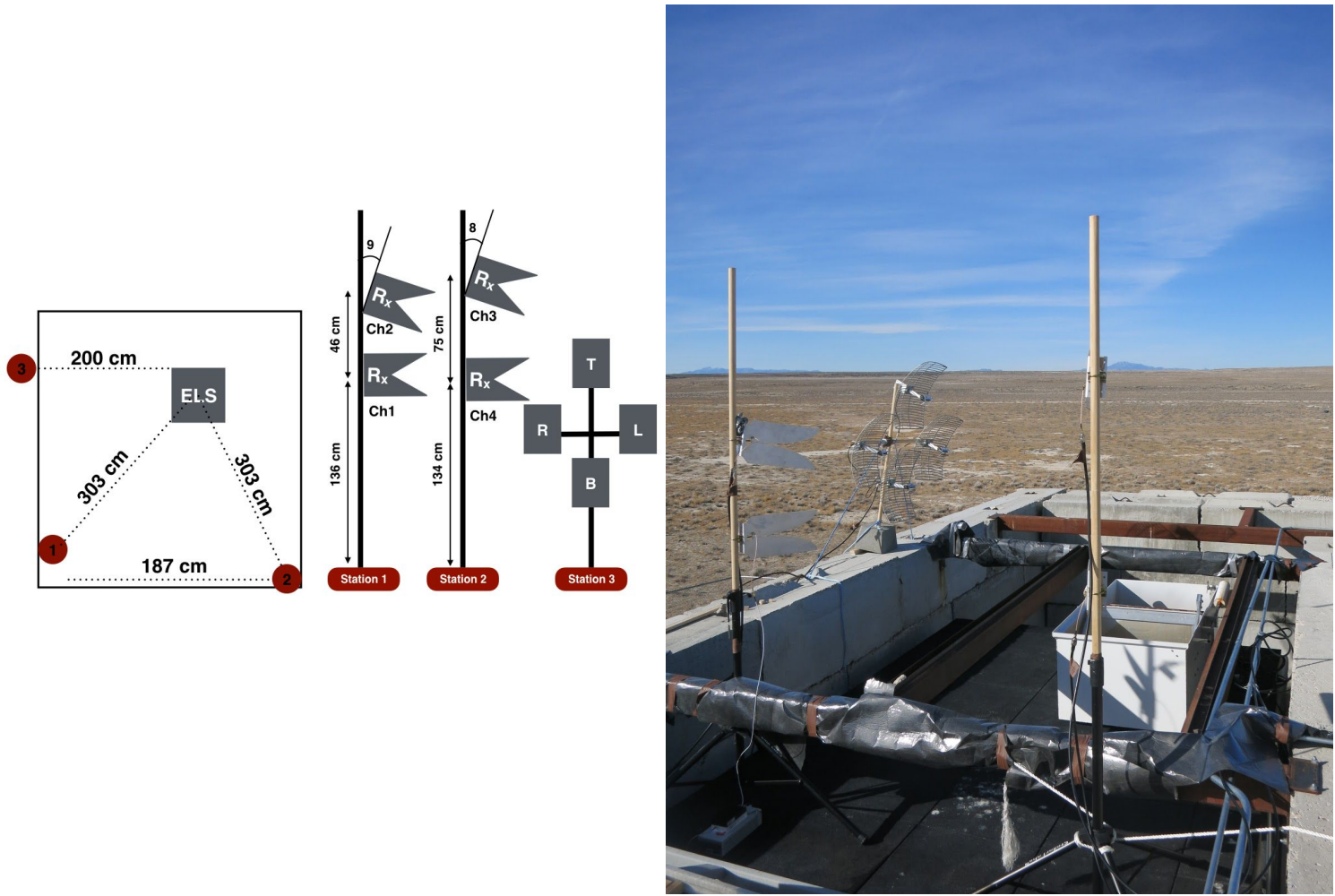

Figure 3: The Vivaldi set-up, consisting out of four Vivaldi antennas and the Wifi-bow antenna set-up.

GSa/s Agilent MSO9254 scope. The fourth Vivaldi receiver antenna was read out without mixing, again after applying a low-pass filter at $2.4 \mathrm{GHz}$. Furthermore, we also read out the signal obtained from one of the Wifi-bow antennas. The beam monitoring was done using an Agilent DSO6104 scope, which reads out the Faraday Cup signal in case it was placed on the beam, as well as the signal from the pick-up coils.

\section{Data}

In this section we give a brief overview of the data that was obtained, and present the first qualitative results. In Fig. 5, we show the raw time-trace measured by the Vivaldi antenna in channel 2 (see Fig. 4 for the details of this channel). Here a strong burst is visible around 2-3 $\mu$ s, which is due to low frequency accelerator noise. The signal is expected around $5 \mu \mathrm{s}$. To investigate a possible radar scatter, we sub-divide the raw time-trace in $124 \mathrm{~ns}$ bins. For each bin, we obtain the frequency content by applying an FFT. In Fig. 6, we show the signal at four different frequencies (50 MHz: top left, $150 \mathrm{MHz}$ : bottom left, $250 \mathrm{MHz}$ : top right, $1550 \mathrm{MHz}$ : bottom right). The signal is plotted for five different transmit powers (orange: $-6 \mathrm{~dB}$; yellow: $-3 \mathrm{~dB}$; green: $0 \mathrm{~dB}$; Blue: $3 \mathrm{~dB}$; Red: $6 \mathrm{~dB}$ ), showing 100 measurements each. The shown data was obtained while transmitting at $1551 \mathrm{MHz}$. At low frequencies, we see the strong noise burst between 2-3 $\mu \mathrm{s}$, which is off-time with the beam leaving the accelerator. Nevertheless, we are interested in the signal observed around $5 \mu$ s, when the beam interacts with the ice. From Fig. 6, it follows that this 


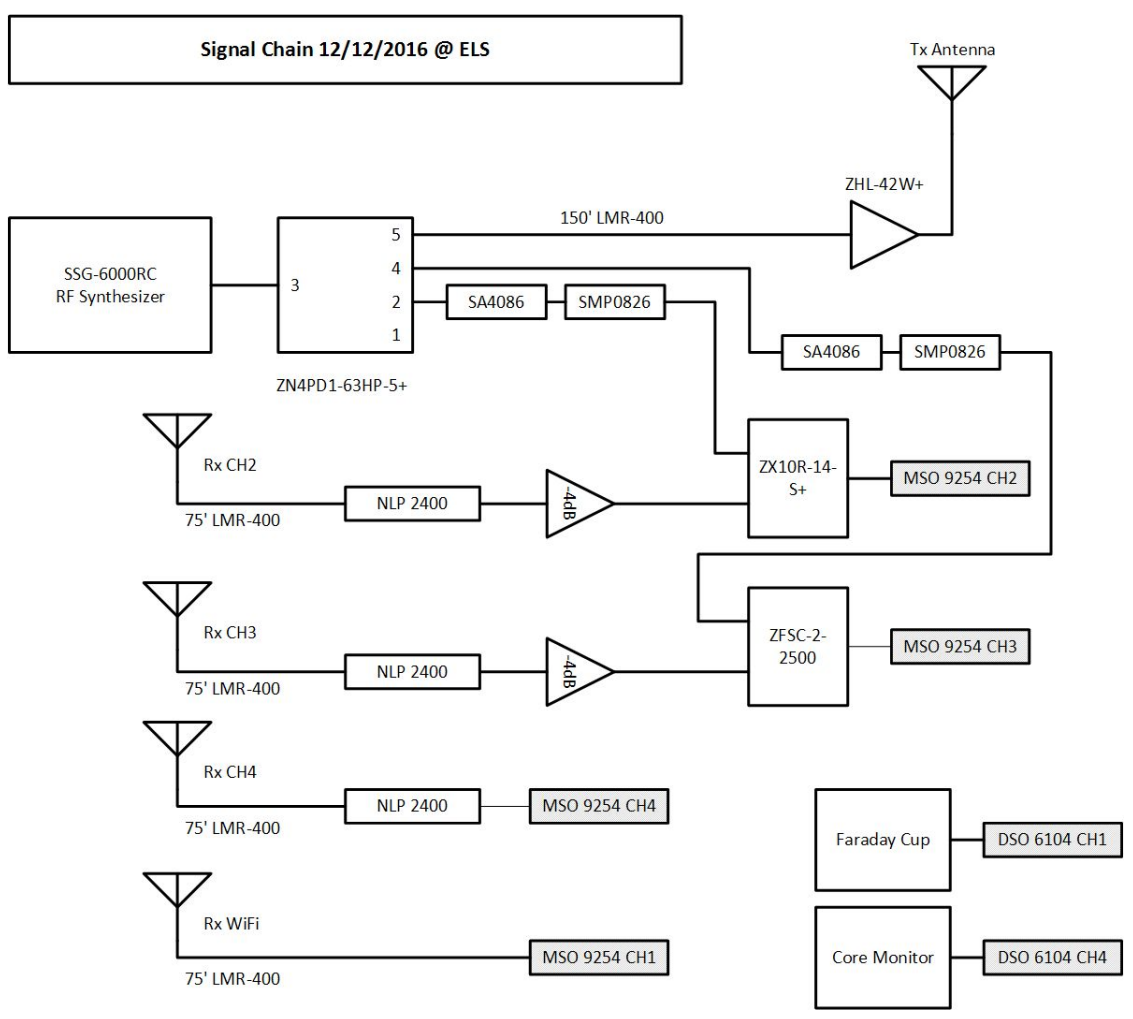

Figure 4: The signal chain for the Vivaldi set-up.

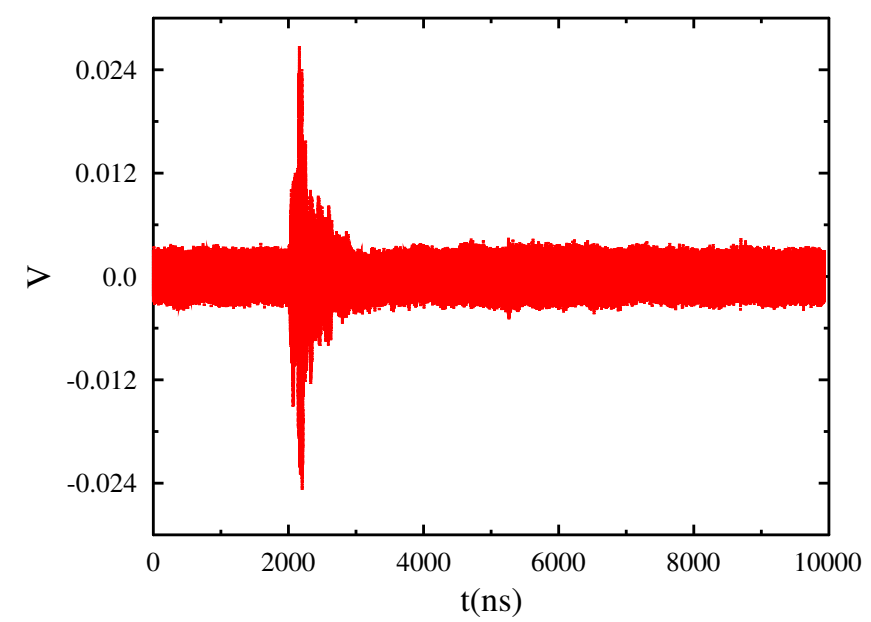

Figure 5: The raw time-trace detected by the Vivaldi antenna in channel 2 of Fig. 3. For this measurement, the beam was directed in to the block of ice, while transmitting at $1551 \mathrm{MHz}$.

signal has the strongest signal-to-noise ratio at $50 \mathrm{MHz}$, and gets weaker while shifting to higher frequencies.

This signal is also observed without transmitter, and can be understood as the beam sudden 

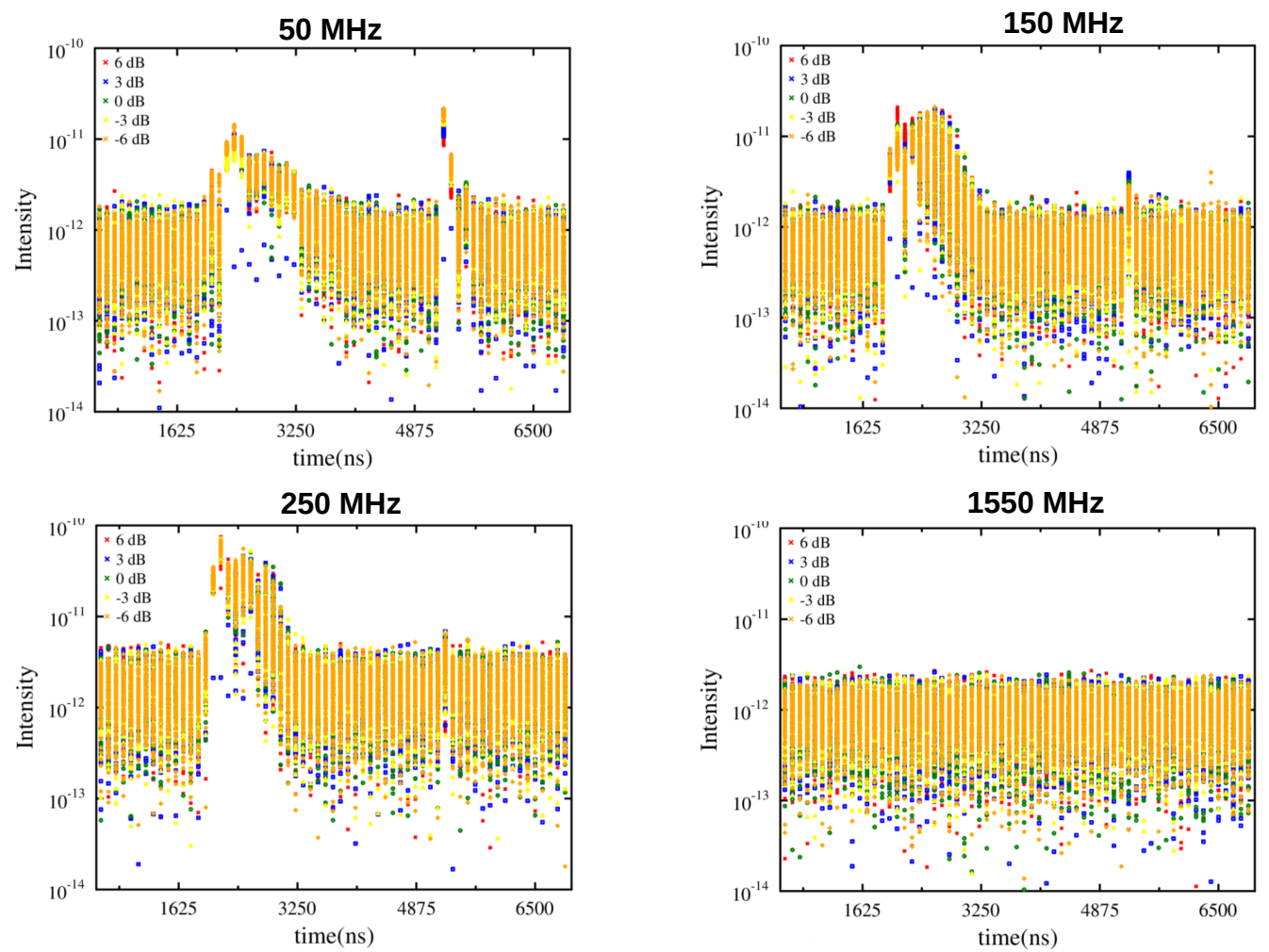

Figure 6: The signal detected at a fixed frequency of $50 \mathrm{MHz}$ : top left, $150 \mathrm{MHz}$ : bottom left, $250 \mathrm{MHz}$ : top right, $1550 \mathrm{MHz}$ : bottom right. The signal is obtained by dividing the raw time trace in $124 \mathrm{~ns}$ time bins, for which the frequency content is determined. The data is shown for five different transmit powers (orange: $-6 \mathrm{~dB}$; yellow: $-3 \mathrm{~dB}$; green: $0 \mathrm{~dB}$; Bleu: $3 \mathrm{~dB}$; Red: $6 \mathrm{~dB}$ ), showing 100 measurements each.

appearance signal, which can be described as an extreme case of coherent transition radiation [13, 14], in combination with the direct Askaryan radio emission [2] from the beam propagation in the ice. Hence, we search for a possible scatter on top of this signal. The most direct signature for a possible radar scatter is the expected scaling of such a signal with the transmit power. Where such a scatter is expected at the transmit frequency [6], the $1550 \mathrm{MHz}$ data does not show any significant signal, nor scaling. At lower frequencies, however, we do see an effect in the data obtained using the Vivaldi antennas. Here the observed signal strength scales (inversely) with input power. At lower transmit frequencies, the LPDA set-up detected the beam sudden appearance signal, but also did not see a significant scatter at the transmit frequency.

\section{Discussion and Conclusion}

We describe a beam test experiment performed at the Telescope Array Electron Light Source facility. This experiment was performed to search for a possible radar signal from a high-energy particle cascade in ice. The obtained data shows a strong transient when the beam exits the accelerator. This signal can be understood as the electron beam sudden appearance signal which can be described as an extreme form of coherent transition radiation. On top of this, we expect the 
Askaryan radio emission from the electron beam interacting with a block of ice which was placed on top of the accelerator.

Besides these signals, we search for a possible radar scatter. The most direct indication for a radar scatter is to search for a signal scaling with the transmit power. Such a power scaling was observed in a previous experiment performed in January 2015 [9]. Here, a power scaling was observed at relatively high frequencies $(1.7 \mathrm{GHz})$, close to the transmit frequency $(1.55 \mathrm{GHz})$. Therefore, this signal was seen as a first hint for a possible scatter. The data obtained in this work, however, does not show any sign of such a scaling close to the transmit frequency. Nevertheless, at lower frequencies, we do observe an (inverse) power scaling. So-far there is no good explanation for this scaling effect, which, if it is due to a radar scatter, is not well described by theory [6]. One indication to explain this effect might be found in the electron beam bunch length, which was narrower for the data obtained in January 2015. This indicates that the observed power scaling is either directly linked to interference between the transmitted signal and the transient sudden appearance and Askaryan signals, or that a possible scatter is coherent over the dimensions of the beam bunch.

\section{Acknowledgments}

The authors would like to thank the TA collaboration for the use of the TA-ELS facility and their indispensable assistance in obtaining the experimental results presented in these proceedings. Furthermore, the authors would like to thank the Chiba University Askaryan Radio Array group for the valuable discussions and the use of their equipment for the ice mounting.

\section{References}

[1] IceCube Collaboration, Science 342 (2013) 1242856

[2] G.A. Askaryan, Sov. Phys. JETP 14 (1962) 441; 21 (1965) 658

[3] P. Allison et al., Astropart. Phys. 35 (2012) 457-477

[4] A. Nelles et al., ARIANNA collaboration, EPJ Web Conf. 135 (2017) 05002

[5] P.W. Gorham et al., Phys. Rev. Lett. 103 (2009) 051103

[6] K.D. de Vries, K. Hanson, T. Meures, Astropart. Phys. 60 (2015) 25-31

[7] P.W. Gorham, Astropart. Phys. 15 (2001) 177

[8] T.Shibata et al., Nucl. Instr. Meth. A 597 (2009) 61-66

[9] K.D. de Vries et al., PoS ( ICRC2015) 1168 (2015)

[10] P. Tinyakov, for the Telescope Array Collaboration, Nucl. Instrum. Meth. A 742 (2014) 29-34

[11] J.B. Verberne et al., Nature 272 (1978) 343-344

[12] M. Kunst, J.M. Warman, Nature 288 (1980) 465-467

[13] K.D. de Vries et al., These Proceedings

[14] K.D. de Vries et al., Astropart.Phys. 74 (2016) 96-104 